\title{
Anesthetic Management During Posterior Spinal Fusion in a Patient With Moyamoya
}

\author{
Mary Jane Romnek ${ }^{\mathrm{a}, \mathrm{d}}$, David P. Martinn, ${ }^{\mathrm{a}, \mathrm{b}}$, Laura Gillc, \\ Jan Klamarc, Joseph D. Tobias ${ }^{\text {a, b }}$
}

\begin{abstract}
Moyamoya disease is an arteriopathy of the vasculature of the central nervous system that predisposes patients to cerebrovascular ischemia and thrombotic strokes. It is characterized by a progressive narrowing of the intracranial component of the internal carotid arteries as well as the proximal branches of the anterior and middle cerebral arteries thereby predisposing patients to episodes of cerebrovascular insufficiency. Moyamoya disease adds an additional level of complexity to the anesthetic care of patients undergoing major surgical procedures related to concerns of maintaining adequate cerebral perfusion and oxygenation. These patients may present with a history of transient ischemic attacks or cerebrovascular accidents with resultant neurological deficits, cognitive delay and seizures at baseline. We present an 18-year-old woman with moyamoya disease who required anesthetic care during a posterior spinal fusion for a neuromuscular disorder. Physiological parameter management intraoperatively is discussed and options for anesthetic care are presented with an emphasis on the use of near infrared spectroscopy to monitor cerebral oxygenation.
\end{abstract}

Keywords: Moyamoya disease; Posterior spinal fusion; Near infrared spectroscopy; Cerebral oxygenation

\section{Introduction}

Moyamoya disease (MMD) is an arteriopathy of the vasculature of the central nervous system (CNS) that predisposes patients to cerebrovascular ischemia and thrombotic cerebrovascular events [1]. It is characterized by a progressive nar-

Manuscript submitted May 3, 2018, accepted May 15, 2018

aDepartment of Anesthesiology \& Pain Medicine, Nationwide Children's Hospital, Columbus, OH, USA

${ }^{b}$ Department of Anesthesiology \& Pain Medicine, The Ohio State University College of Medicine, Columbus, OH, USA

'Department of Orthopedic Surgery, Nationwide Children's Hospital and The Ohio State University College of Medicine, Columbus, OH, USA

${ }^{\mathrm{d} C}$ Corresponding Author: Mary Jane Romnek, Department of Anesthesiology \& Pain Medicine, Nationwide Children's Hospital, 700 Children's Drive, Columbus, OH 43205, USA.

Email: MaryJane.Romnek@nationwidechildrens.org

doi: https://doi.org/10.14740/jmc3072w rowing or stenosis of the intracranial component of the internal carotid arteries as well as the proximal branches of the anterior and middle cerebral arteries [1,2]. Collateral circulation from branches of the intracranial carotid artery creates the characteristic angiographic appearance that gives the disease its name. The characteristic angiographic appearance was called "moyamoya" after the Japanese expression for "something hazy just like a puff of cigarette smoke drifting in the air" [3]. Although once believed to be specific to the Japanese population, it has more recently been reported in patients of various other ethnic groups including those with specific comorbid conditions including hemoglobin disorders such as sickle cell disease [4]. Given the multi-organ involvement of the disorder, comorbid conditions may arise that require surgical intervention. We present an 18-year-old woman with MMD who required anesthetic care during a posterior spinal fusion for neuromuscular scoliosis. Physiological parameter management intraoperatively is discussed and options for anesthetic care are presented with an emphasis on the use of near infrared spectroscopy (NIRS) to monitor cerebral oxygenation [5].

\section{Case Report}

Institutional Review Board approval is not required for publication of isolated case reports at Nationwide Children's Hospital (Columbus, OH, USA). An 18-year-old woman with MMD and a history of left middle cerebral artery (MCA) infarction in the remote past presented for a posterior spinal fusion (PSF) with instrumentation, allograft placement and pelvic fixation due to severe neuromuscular scoliosis and hip subluxation. Other medical history included cerebral palsy with spastic quadriparesis, seizures, mixed receptive-expressive language disorder, asthma, gastric-tube dependence and sickle cell disease status after bone marrow transplantation. Prior anesthetic issues included difficult peripheral intravenous access, slow emergence, perioperative oxygen desaturations, and postoperative nausea and vomiting. She had no exposure to secondhand smoke, immunizations were up to date and there were no allergies. A recent electrocardiogram and echocardiogram were unremarkable. Chronic medications included inhaled fluticasone ( 2 puffs twice a day) and inhaled albuterol ( 2 puffs every $4 \mathrm{~h}$ as needed), docusate sodium (50 $\mathrm{mg}$ daily), zolpidem (10 $\mathrm{mg}$ at bedtime), lacosamide (170 $\mathrm{mg}$ twice a day), glycopyrrolate (1 mg twice a day), levetiracetam $(1,600 \mathrm{mg}$ twice a day), folic acid (1 $\mathrm{mg}$ once a day), aspirin ( $81 \mathrm{mg}$ once 
a day), cholecalciferol (400 units once a day) and diazepam (10 $\mathrm{mg}$ rectally as needed for seizures lasting longer than 5 min). In the pre-anesthesia assessment clinic, blood could not be obtained due to extreme difficulty obtaining venous access. Therefore, laboratory values including baseline hemoglobin and hematocrit as well as the type and cross were deferred until the day of surgery. On the day of surgery, the patient was in her normal state of health and physical examination was unremarkable aside from developmental delay and spasticity. The patient was held nil per os for $4 \mathrm{~h}$ for clear liquids and 8 $\mathrm{h}$ for solids except for her usual morning anticonvulsant medications. The patient was taken to the operating room and was placed on standard American Society of Anesthesiologists' monitors while on the stretcher. In addition, bilateral cerebral oximeter probes (NIRS) were placed while the patient was on room air and prior to anesthetic induction with baseline measurements obtained [5]. Baseline values while breathing room air were 83 on the left and 77 on the right. Additionally, depth of anesthesia was monitored using the bispectral index (BIS). Inhalation induction was performed with incremental concentrations of sevoflurane in $100 \%$ oxygen and a 20 -gauge intravenous (IV) catheter was placed in the right hand. Propofol (50 $\mathrm{mg})$, rocuronium $(30 \mathrm{mg})$ and midazolam $(2 \mathrm{mg})$ were administered. Direct laryngoscopy was performed with a Macintosh 3 blade and a 7.0 cuffed oral endotracheal tube was placed with a grade 1 view. A 14-gauge IV catheter was placed in the right basilic vein with ultrasound guidance and baseline laboratory values were obtained including a type and crossmatch. An arterial catheter was then inserted into the left radial artery in the forearm using ultrasound guidance. Due to the patient's spasticity, a traditional radial artery catheter was not feasible, so the artery was accessed more proximally. Given the patient's history of difficult IV access and expected longterm hospital stay, a 5 French, $12 \mathrm{~cm}$, double-lumen central venous catheter was then placed in the right internal jugular vein under ultrasound guidance. The neuromonitoring technicians then placed monitoring devices for motor (MEP) and somatosensory (SSEP) evoked potentials. Using a prone foam pillow, the patient was turned prone and placed on the Jackson table with the assistance of the surgical team. Extreme care was taken to ensure that all pressure points were padded and the patient was in as neutral a position as her body would allow. To facilitate neurophysiological monitoring, maintenance of anesthesia included desflurane (0.5 - 0.6 minimum alveolar concentration) titrated to maintain the BIS at $50-60$ and a remifentanil infusion $(0.1-0.3 \mu \mathrm{g} / \mathrm{kg} / \mathrm{min})$ [6]. Phenylephrine was used to maintain the mean arterial pressure (MAP) and NIRS values at baseline with an MAP in the range of $75-85$ $\mathrm{mmHg}$. Throughout the course of the posterior spinal fusion, the NIRS monitor was used to ensure adequate cerebral oxygenation and guide MAP, ventilation and transfusion therapy. Decreases in the NIRS values corresponded with both blood pressure changes and decreases in the hemoglobin concentration. Throughout the case, the NIRS was maintained bilaterally at $\geq 60$. At the end of the case, estimated blood loss was 2,200 $\mathrm{mL}$. Fluid and blood product therapy included 1,396 mL (4 units) of packed red blood cells (RBCs), 3,525 mL of cell saver blood, $315 \mathrm{~mL}$ (1 unit) of fresh frozen plasma (FFP), $201 \mathrm{~mL}$ (1 unit) of pheresed platelets, $6,000 \mathrm{~mL}$ of crystal- loid and $1,750 \mathrm{~mL}$ of $5 \%$ albumin. Postoperatively, the patient was transported to the pediatric intensive care unit (ICU). Her trachea was extubated a few hours later that same evening. Postoperative pain control was provided by nurse-controlled analgesia with hydromorphone and intermittent, fixed interval dosing of intravenous acetaminophen. She recovered well and was discharged from the hospital on postoperative day 8 .

\section{Discussion}

Moyamoya disease was originally described in Japan, but more recent epidemiological studies note that the disease is found across all ethnicities, with an increased incidence in the Eastern compared to the Western hemispheres [7]. Although the incidence varies depending on the country, people of Asian descent tend to have an incidence of MMD similar to that noted in Japan regardless of their country of origin [7]. The female to male ratio is 1.8 and the overall prevalence in Japan is 3.16 - 10.5 per 100,000 [7, 8]. Patients with MMD disease typically present during two distinct age ranges: the first being $5-10$ years of age and the second being 40 - 50 years of age [7]. Children typically present with ischemic events resulting in hemiparesis, sensory impairment or aphasia/dysarthria, while adults present with intracranial hemorrhage due to rupture of the collateral vessels that tend to be more fragile [7]. There is an additional subset of patients who are asymptomatic, but found to have the classical angiographic appearance of MMD on MRI/MRA [7-9]. There appears to be a genetic factor to the development of MMD involving the susceptibility gene ring finger protein 213 (RNF213) located on chromosome 17, although the pathway from this abnormality to the development of MMD is unknown [7].

Treatment options include medical management with aspirin to help prevent thromboembolic events as well as surgical management via pial synangiosis [7, 8]. Management options for asymptomatic patients are currently under prospective investigation in an ongoing clinical trial [9]. Surgical management with pial synangiosis can be performed via an indirect revascularization with tissues containing the external carotid artery (ECA), including dura mater, temporal muscle, galeal tissue or superficial temporal artery, being placed on the brain with resulting enhancement of angiogenic factors from the ischemic brain $[7,8]$. It can also be performed more directly by attaching the superficial temporal artery directly to the anterior cerebral artery or middle cerebral artery [7]. The latter provides immediate improvement of cerebral hemodynamic status, compared to the former which requires neovascularization requiring approximately 3 months [7]. Pediatric patients with MMD have a higher risk of perioperative stroke when the indirect bypass technique is used; therefore, the surgical management may vary depending on the age of the patient [10].

Given the prominent CNS involvement of the disease process, surgical interventions are frequently required to correct the sequelae including neuromuscular scoliosis as was seen in our patient. General perioperative concerns are addressed during the preoperative visit. The presence of contractures and the history of multiple surgical procedures may lead to difficul- 
ties with vascular access as noted in our patient. Both arterial and central venous accesses were planned in our patient during this major surgical intervention to facilitate intraoperative and postoperative care and monitoring. Clinical experience has suggested the utility of ultrasound in facilitating central venous as well as peripheral venous and arterial access [11]. Additionally, these patients may have an underlying seizure disorder related to previous strokes or episodes of cerebral ischemia. Routine anticonvulsant medications should be administered the day or surgery with ongoing intraoperative dosing [12]. In specific cases, alternatives may be required perioperatively for anticonvulsant medications that require oral administration. As noted previously, ongoing therapy with aspirin is frequently used to prevent stroke recurrence. For major surgical procedures with bleeding risk, this therapy should be held for 5 - 7 days prior to surgery to avoid effects on platelet function. Although our general practice for posterior spinal fusion includes the intraoperative administration of tranexamic acid to limit intraoperative blood loss, given the theoretical concerns of thrombosis, no anti-fibrinolytic agents were administered.

During these major surgical procedures, alterations in ventilation, fluid status and myocardial function may alter the primary determinants of cerebral oxygen delivery thereby predisposing these patients to further deterioration of their CNS status related to ischemia [11]. The primary goals for perioperative management include attention to parameters which control cerebral perfusion pressure and oxygen delivery including MAP, hemoglobin concentration and the arterial partial pressure of carbon dioxide $\left(\mathrm{PaCO}_{2}\right)$. While hypocapnia clearly decreases cerebral blood flow, hypercapnia may dilate only the normal vasculature with an inadequate response in diseased vessels resulting in a steal phenomenon. Therefore, current recommendations including avoidance of hypocapnia or hypercapnia with maintenance of normocarbia during intraoperative and postoperative care [13-15]. The potential impact of $\mathrm{PaCO}_{2}$ is illustrated by several reports. In a retrospective review of 15 patients, Samagh et al reported that patients with mean intraoperative end tidal carbon dioxide $\left(\mathrm{ETCO}_{2}\right)$ values either less than $31 \mathrm{~mm} \mathrm{Hg}$ or more than $35 \mathrm{~mm} \mathrm{Hg}$ had a statistically significant prolongation of hospital stay [14]. Changes in $\mathrm{PaCO}_{2}$ may impact the postoperative course as anecdotal and retrospective evidence demonstrates the potential for cerebral ischemia to develop postoperatively related to hyperventilation associated with agitation and crying [14-16]. In a review of 169 EDAS procedures in children, Matsushima et al reported cerebral infarction in 6 patients $(3.6 \%) 6$ patients, which were attributed to hyperventilation associated with crying during the postoperative period [17]. These issues mandate close monitoring of neurological status in an ICU setting postoperatively with ongoing control of physiological parameters that regulate cerebral blood including $\mathrm{PaCO}_{2}, \mathrm{MAP}$ and hemoglobin concentration. Adequate pain control is essential to avoid agitation and crying related to pain or anxiety [13, 14]. Nomura et al demonstrated the efficacy of protocols to treat pain and prevent crying with hyperventilation [16]. When comparing 14 surgical cases prior to these protocols with 11 after the protocols were introduced, they noted a decreased incidence of transient ischemic attacks from $28.6 \%$ to $3.7 \%$ and a decrease in the average postoperative hospital stay from 21.3 days to 16.1 days.

In general, there is no clear evidence to suggest the advantage of any particular anesthetic technique or specific agents for the induction and maintenance of anesthesia in patients with MMD. Our technique was chosen based on our usual clinical practice to facilitate neurophysiological monitoring of spinal cord integrity [6]. Successful outcomes have been reported with both intravenous-based (total intravenous anesthesia or TIVA) and volatile-based techniques. The most important perioperative goals remain the close control or physiological parameters that control cerebral perfusion with maintenance of MAP, hemoglobin and normocapnia. Given the potential for the intraoperative alterations in the physiological factors which affect cerebral oxygenation related to anesthetic and surgical care, we suggest that the NIRS monitor can be used to assist in maintaining adequate cerebral perfusion especially during surgical cases where intraoperative fluctuations of physiological parameters are frequent. With such caveats in mind maintenance of adequate cerebral perfusion can be facilitated to improve the perioperative outcomes of patients with MMD.

\section{References}

1. Scott RM, Smith ER. Moyamoya disease and moyamoya syndrome. N Engl J Med. 2009;360(12):1226-1237.

2. Scott RM, Smith JL, Robertson RL, Madsen JR, Soriano $\mathrm{SG}$, Rockoff MA. Long-term outcome in children with moyamoya syndrome after cranial revascularization by pial synangiosis. J Neurosurg. 2004;100(2 Suppl Pediatrics):142-149.

3. Suzuki J, Takaku A. Cerebrovascular "moyamoya" disease. Disease showing abnormal net-like vessels in base of brain. Arch Neurol. 1969;20(3):288-299.

4. Steven A, Raghavan P, Rath TJ, Gandhi D. Neurologic and Head and Neck Manifestations of Sickle Cell Disease. Hematol Oncol Clin North Am. 2016;30(4):779798.

5. Tobias JD. Cerebral oxygenation monitoring: nearinfrared spectroscopy. Expert Rev Med Devices. 2006;3(2):235-243.

6. Martin DP, Bhalla T, Thung A, Rice J, Beebe A, Samora $\mathrm{W}$, Klamar J, et al. A preliminary study of volatile agents or total intravenous anesthesia for neurophysiological monitoring during posterior spinal fusion in adolescents with idiopathic scoliosis. Spine (Phila Pa 1976). 2014;39(22):E1318-1324.

7. Guey S, Tournier-Lasserve E, Herve D, Kossorotoff M. Moyamoya disease and syndromes: from genetics to clinical management. Appl Clin Genet. 2015;8:49-68.

8. Digiusto M, Bhalla T, Grondin R, Tobias JD. Perioperative care of the pediatric patient for pial synangiosis surgery. Int J Clin Exp Med. 2013;6(3):231-238.

9. Kuroda S, AMORE Study Group. Asymptomatic moyamoya disease: literature review and ongoing AMORE study. Neurol Med Chir (Tokyo). 2015;55(3):194-198.

10. Hishikawa T, Sugiu K, Date I. Moyamoya disease: a review of clinical research. Acta Med Okayama. 
2016;70(4):229-236.

11. Jones CT, Raman VT, DeVries S, Cole JW, Kelleher KJ, Tobias JD. Optimizing anticonvulsant administration for children before anesthesia: a quality improvement project. Pediatr Neurol. 2014;51(5):632-640.

12. Tobias JD, Martin DP, Bhalla T. Ultrasound for central venous, arterial, and peripheral venous cannulation in the pediatric population. Pediatr Anesth Crit Care J. 2014;2:93-101.

13. Baykan N, Ozgen S, Ustalar ZS, Dagcinar A, Ozek MM. Moyamoya disease and anesthesia. Paediatr Anaesth. 2005;15(12):1111-1115.

14. Samagh N, Bhagat H, Grover VK, Sahni N, Agarwal A, Gupta SK. Retrospective analysis of perioperative fac- tors on outcome of patients undergoing surgery for Moyamoya disease. J Neurosci Rural Pract. 2015;6(2):262265.

15. Parray T, Martin TW, Siddiqui S. Moyamoya disease: a review of the disease and anesthetic management. J Neurosurg Anesthesiol. 2011;23(2):100-109.

16. Nomura S, Kashiwagi S, Uetsuka S, Uchida T, Kubota H, Ito $\mathrm{H}$. Perioperative management protocols for children with moyamoya disease. Childs Nerv Syst. 2001;17(45):270-274.

17. Matsushima Y, Aoyagi M, Suzuki R, Tabata H, Ohno $\mathrm{K}$. Perioperative complications of encephalo-duro-arterio-synangiosis: prevention and treatment. Surg Neurol. 1991;36(5):343-353. 